\title{
Experimental Study of Stacked Rectangular Microstrip Antenna for Dual-Band
}

\author{
Rajesh Kumar Vishwakarma ${ }^{1}$, Sanjay Tiwari ${ }^{2}$ \\ School of Studies in Electronics, Pt. Ravishankar Shukla University, Raipur, Chhattisgarh (C.G) \\ E-mail:rkv_786@yahoo.com,drsanjaytiwari@gmail.com \\ Received August 27, 2009; revised September 14, 2009; accepted September 20, 2009
}

\begin{abstract}
A dual-band characteristic of stacked rectangular microstrip antenna is experimentally studied. It is a probe fed antenna for impedance matching with $50 \Omega$ coaxial cable. This antenna works well in the frequency range ( 2.86 to $4.63 \mathrm{GHz}$ ). It is basically a low cost, light weight medium gain antenna, which is used for mobile communication. The variations of the length and width $(1 \mathrm{~mm})$ of the stacked rectangular patch antenna have been done. And it is found dual resonance with increasing lower resonance frequency and almost constant upper resonance frequency with increases of the length \& width of rectangular microstrip antenna. The input impedance and VSWR, return loss have been measured with the help of Network analyzer.
\end{abstract}

Keywords: Microstrip Antenna, Stacked Patch Antenna, Dual-Band Antenna, Network Analyzer, Bandwidth

\section{Introduction}

The demand for application of microstrip antenna in various communication systems has been increasing rapidly due to its lightweight, low cost, small size, ease of integration with other microwave components [1-4]. Microstrip antenna gained in popularity and become a major research topic in both theoretically and experimentally. However one of main disadvantages of microstrip antenna is their narrow band width. It is well known that the multilayer structure is useful method to improve these problems. The researcher have investigated their basic characteristics and extensive efforts have also been developed to design of electromagnetically coupled two layer elliptical microstrip stacked antenna [5], stacked square patch antenna for Bluetooth application [6] and analysis of stacked microstrip rectangular microstrip antenna [7]. Several methods have been presented in the last years to improve it's such as: thicker substrate [8] reactive matching network [9], and stacked patches [10-12]. Microstrip patch antenna elements with a single feed are used in many popular for various radar and communication system such as synthetics aperture radar (SAR), dual-band, multi-band, mobile communication system and Global Positing Systems (GPS) [13]. It may be mentioned that the bandwidth can also be improved by stacking a parasitic patch on the fed patch [14]. Therefore in this present paper, we observed on an electromagnetically stacked rectangular microstrip antenna with number of parasitic elements. By using two stacked patches with the wall at edges between the two patches, one can obtained enhance impedance bandwidth. Experimental work is carried out to study the effect of stacking on various parameters of antenna.

\section{Antenna Design Calculation}

The transmission line model is used to design rectangular microstrip antenna which is stacked one by one. Rectangular microstrip antenna (RMA) is designed by using following procedures.

\subsection{Patch Width and Patch Length}

The first to design the patch is choosing a suitable dielectric substrate of suitable thickness. For rectangular microstrip antenna, the width $\mathrm{W}$ and the length $\mathrm{L}$ depends on the resonant frequency $f_{r}$ and the parameters of the substrate employed [1].

To design the rectangular patch width of the antenna is given by

$$
W=\frac{c}{2 \cdot f_{r}}\left[\left(\frac{\varepsilon_{r}+1}{2}\right)\right]^{-\frac{1}{2}}
$$

where $\mathrm{C}=$ velocity of light

$W=$ width of the microstrip patch

$\varepsilon_{r}=$ Dielectric constant of the substrate Length of the resonant element is given by 


$$
L=\frac{c}{2 \cdot f_{r} \sqrt{\varepsilon_{e f f}}}-2 \Delta l
$$

where

$\varepsilon_{\text {eff }}=$ Effective dielectric constant of the substrate

$\Delta l=$ Line extension

$\varepsilon_{\text {eff }}$ and $\Delta l$ can be expressed as

$$
\begin{aligned}
& \varepsilon_{\text {eff }}=\left(\frac{\varepsilon_{r}+1}{2}\right)+\left(\frac{\varepsilon_{r}-1}{2}\right) \cdot\left(1+\frac{10 \cdot h}{W}\right)^{-0.5} \\
& \Delta l=0.412 h\left[\frac{0.262+\frac{W}{h}}{0.813+\frac{W}{h}}\right]\left[\frac{\varepsilon_{e f f}+0.3}{\varepsilon_{\text {eff }}-0.258}\right]
\end{aligned}
$$

where, $h$ is the thickness of the substrate.

The driven patches were designed to operate at a resonant frequency of $3.0 \mathrm{GH}_{\mathrm{z}}$, their length and width were calculated to be $L=23.01 \mathrm{~mm}$ and width $W=30.01 \mathrm{~mm}$ respectively. The remaining five patches were designed with the differences of $1 \mathrm{~mm}$ in the length and width with increasing mode. They are stacked in the manner shown in (Figure 1).

\subsection{Feeding Design}

The feeding point was designed for lower patch to match the $(50 \Omega)$ coaxial cable feed and the patch. The feeding point was fabricated along the length of the patch. The input conductance of the patch fed on the edge slot will be twice the conductance of one of the edge slots as suggested by Harrington and given by [15].

$$
G=\frac{\pi W}{\eta \lambda_{0}}\left[1-\frac{(k h)^{2}}{24}\right]
$$

where $\eta=120 \pi \quad k=\frac{2 \pi}{\lambda_{0}} \quad \lambda_{0}=$ Wavelength of free space

So the input impedance of the patch is given by

$R_{l}=1 / 2 G(\Omega)$, where $\mathrm{G}$ is radiation conductance.

So the upper patch is parasitic and the lower patch is fed with a co axial probe at the position $\left(X_{0}, Y_{0}\right)$ where $X_{0}=$
$6.40 \mathrm{~mm}$ and $Y_{0}=15.725 \mathrm{~mm}$. All the designed patches have been stacked one over the driven patch.

\section{Experimental Measurements}

The rectangular microstrip antenna was measured using network analyzer [Agilent E8363B A.04.06]. For obtaining the desired dual-frequency behavior the lower patch conductor of the coaxial feed is allowed to pass through a clearance hole in the lower patch and is then eclectically connected to the upper patch as shown in (Figure 1). The rectangular microstrip consists of one driven patch and five parasitic patches designed for 3.0 $\mathrm{GH}_{\mathrm{z}}$. The variation of length and width with design frequencies of antennas are shown in the Table 1. The

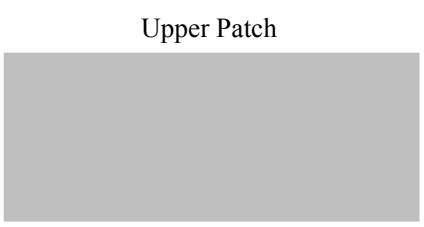

$\mathrm{L}_{1}$

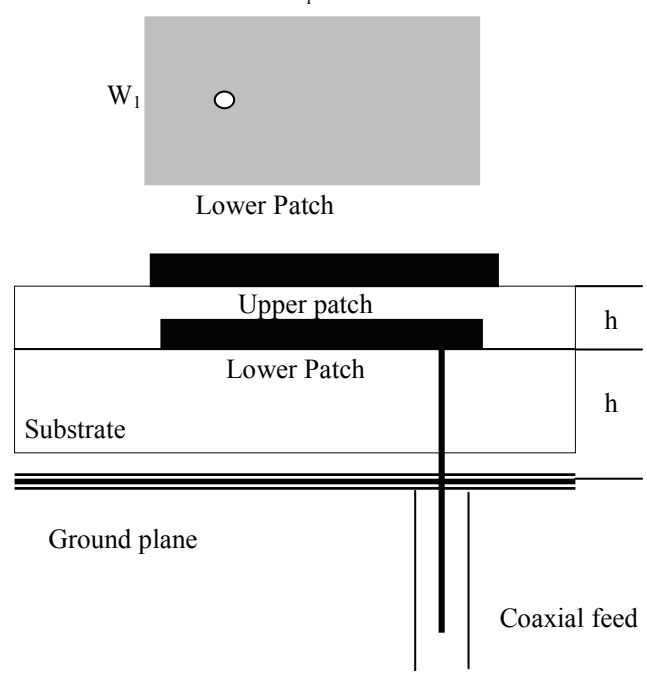

Figure 1. The dual-band electromagnetically coupled stacked antenna. (a) Upper patch and Lower patch; (b) Cross section.

Table 1. variation of length and width with design frequencies of patches.

\begin{tabular}{ccc}
\hline Patches in $\mathrm{mm}$ & Dimension of patch Length and width $(\mathrm{mm})$ & Design frequency in GHz \\
\hline $\begin{array}{c}\text { Antenna } \\
\text { (Driven patch) }\end{array}$ & $23.01 \times 30.15$ & 3.0 \\
Patch 1 & $24.01 \times 31.15$ & 3.0 \\
Patch 2 & $25.01 \times 32.15$ & 3.0 \\
Patch 3 & $26.01 \times 33.15$ & 3.0 \\
Patch 4 & $27.01 \times 34.15$ & 3.0 \\
Patch 5 & $28.01 \times 35.15$ & 3.0 \\
\hline
\end{tabular}


Table 2. Variation of upper and lower resonance frequencies and their ratio $\left(f_{2} / f_{1}\right)$ with return loss in $\mathrm{dB}$.

\begin{tabular}{ccccc}
\hline $\begin{array}{c}\text { Patches } \\
\text { in mm }\end{array}$ & $\begin{array}{c}\text { Lower } \\
\text { resonance frequency } \\
\mathrm{f}_{1}(\mathrm{GHz})\end{array}$ & $\begin{array}{c}\text { Upper } \\
\text { resonance frequency } \\
\mathrm{f}_{2}(\mathrm{GHz})\end{array}$ & $\begin{array}{c}\text { Ratio of resonance } \\
\text { frequencies } \\
\mathrm{f}_{2} / \mathrm{f}_{1}(\mathrm{GHz})\end{array}$ & $\begin{array}{c}\text { Return loss }(\mathrm{dB}) \\
\text { for } \mathrm{f}_{1}(\mathrm{GHz})\end{array}$ \\
\hline Patch 1 & 2.868 & 4.585 & 2.868 & $-\begin{array}{c}\text { Return loss }(\mathrm{dB}) \\
\text { for } \mathrm{f}_{2}(\mathrm{GHz})\end{array}$ \\
Patch 2 & 2.904 & 4.625 & 2.904 & -11.85 \\
Patch 3 & 2.903 & 4.593 & 2.903 & -10.89 \\
Patch 4 & 2.917 & 4.624 & 2.917 & -13.26 \\
Patch 5 & 2.917 & 4.636 & 2.917 & -13.80 \\
\hline
\end{tabular}

variation of upper and lower resonance frequencies and their ratio $\left(f_{2} / f_{1}\right)$ with increasing patch length and width are shown in the Table 2 . In order to study the performance of the development rectangular microstrip antenna return loss, resonance frequencies and VSWR were measured experientially with different number of parasitic antenna shown in the Table 2 . The broad band width is achieved about $45 \%$ with the stacking of the antenna.

\section{Design Parameters}

The various design parameters of the antenna are as follows:

Substrate material used Glass Epoxy

Thickness of the dielectric substrate $h=1.59 \mathrm{~mm}$

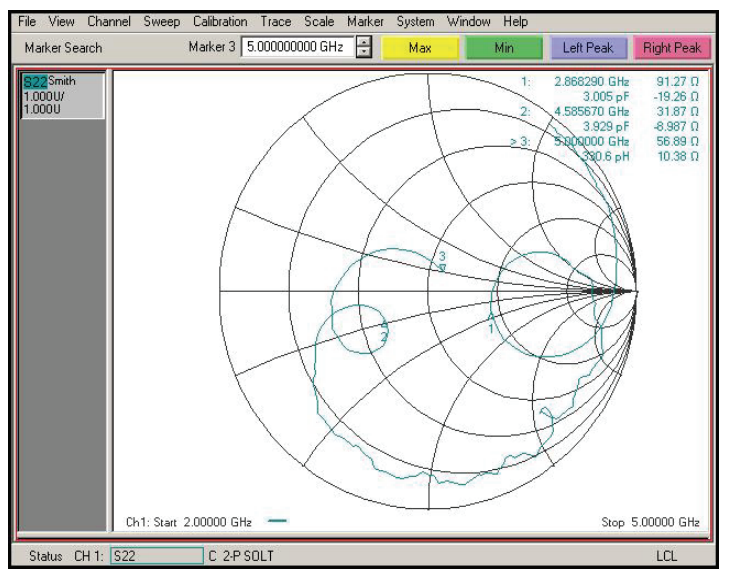

(a)

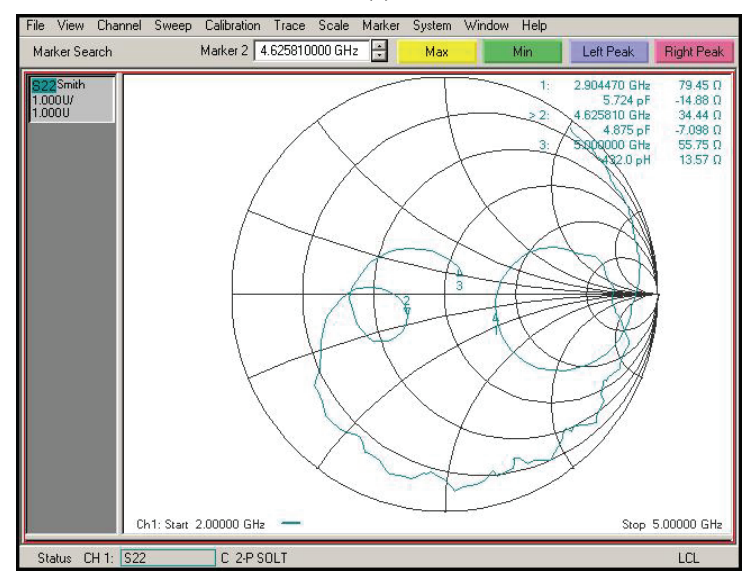

(b)

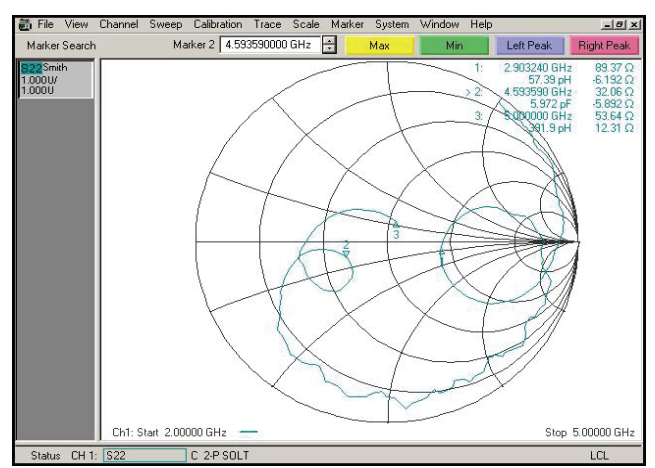

(c)

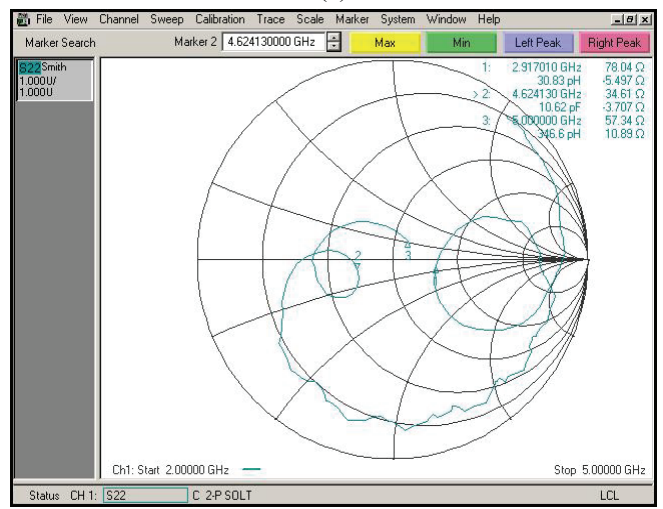

(d)

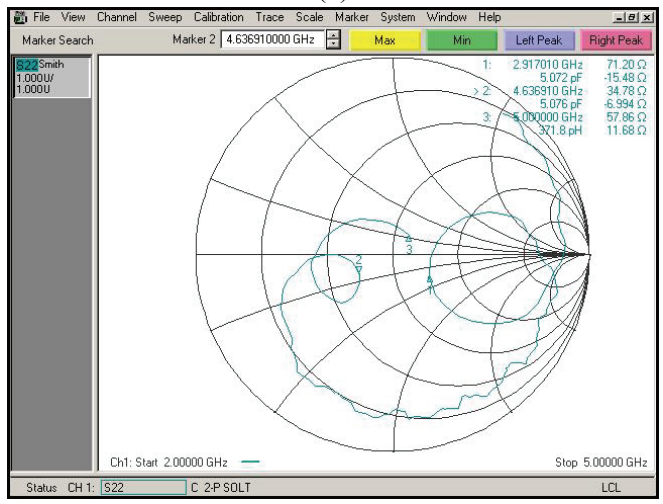

(e)

Figure 2. (a) Variations of input impedance with frequency for patch dimension 24.01x 31.15; (b) Variations of input impedance with frequency for patch dimension $25.01 \times 32.15$; (c) Variations of input impedance with frequency for patch dimension 26.01x 33.15; (d) Variations of input impedance with frequency for patch dimension 27.01x 34.15; (e) Variations of input impedance with frequency for patch dimension 28.01x 35.15. 


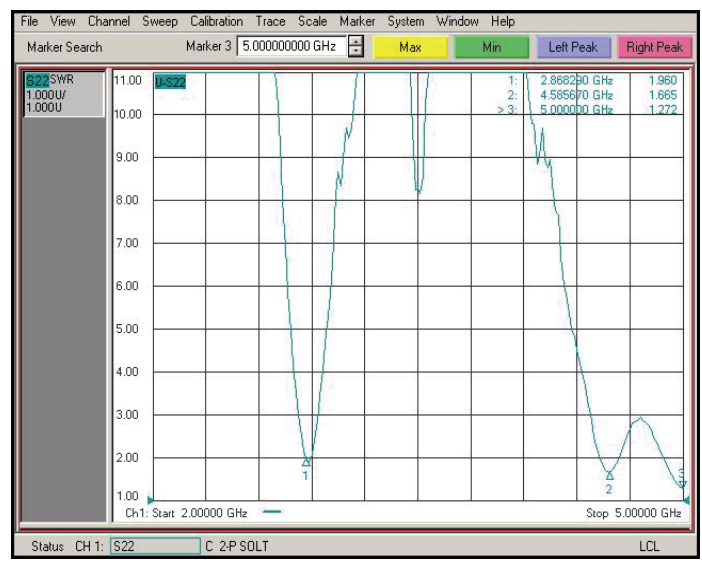

(a)

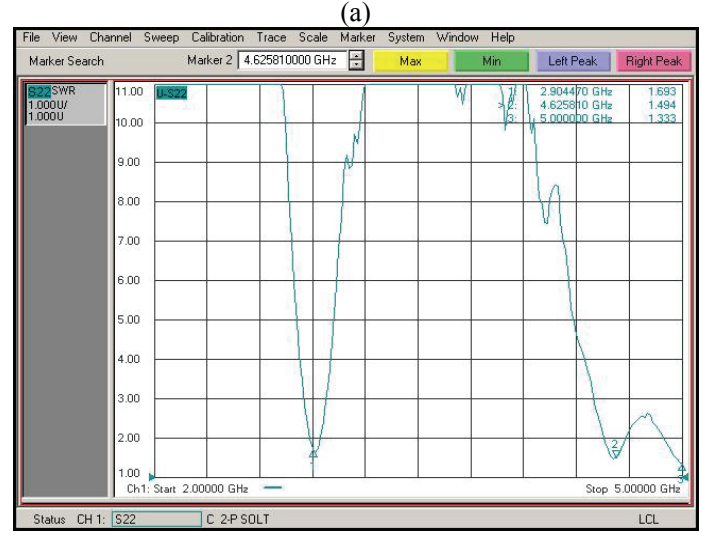

(b)

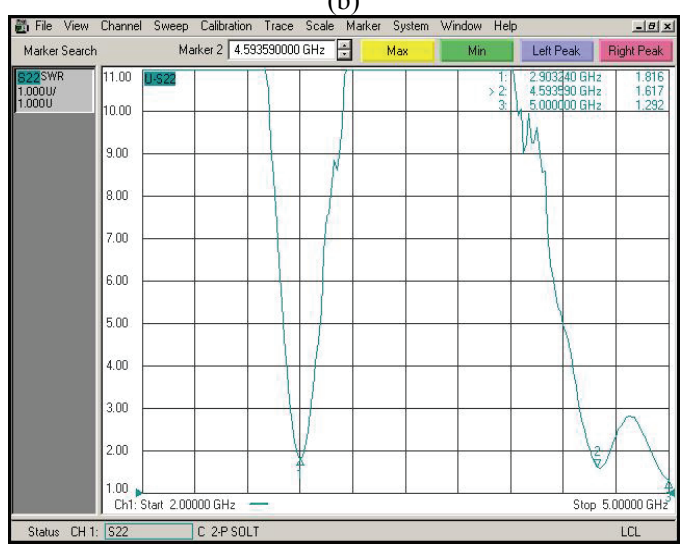

(c)

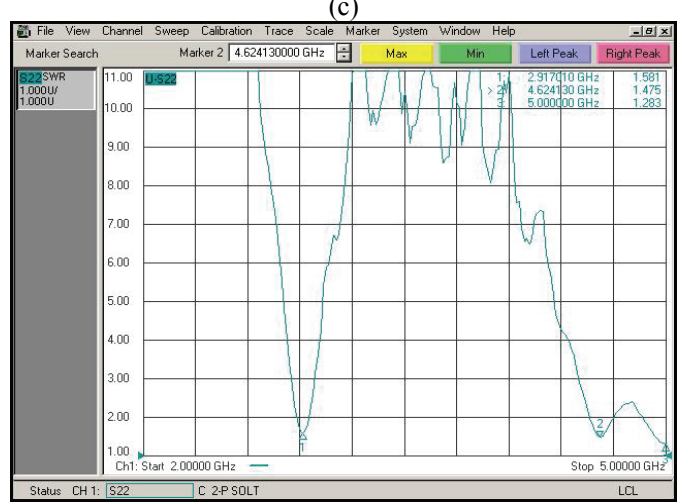

(d)

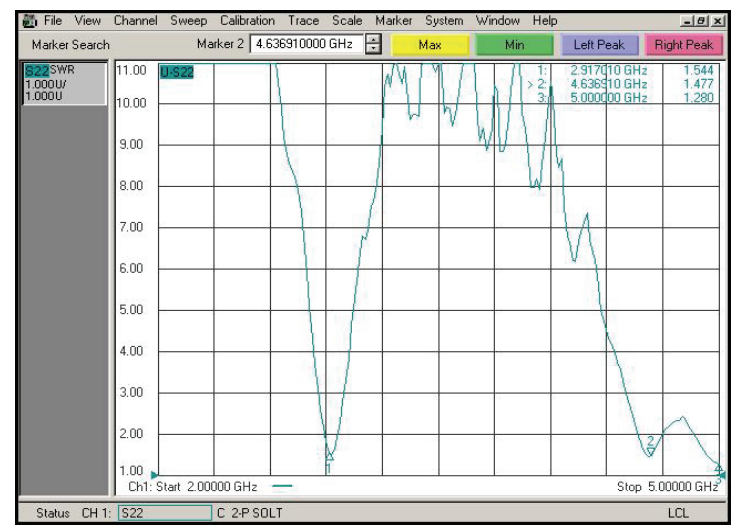

(e)

Figure 3. (a) Variations of VSWR with frequency for patch dimension 24.01x 31.15; (b) Variations of VSWR with frequency for patch dimension 25.01x 32.15; (c) Variations of VSWR with frequency for patch dimension 26.01x 33.15; (d) Variations of VSWR with frequency for patch dimension 27.01x 34.15; (e) Variations of VSWR with frequency for patch dimension $28.01 \times 35.15$.

Relative permittivity of the substrate $\varepsilon_{r}=4$.

Design frequency $f=3.0 \mathrm{GHz}$

Thickness of the patch $t=0.0018 \mathrm{~cm}$

And designed values were calculated using the standard equations, which are given below.

The width of the rectangular patch (Driven Patch) $W_{1}=$ $30.15 \mathrm{~mm}$.

The length of the rectangular patch (Driven Patch) $L_{1}$ $=23.01 \mathrm{~mm}$.

The variation of length of the patch (Parasitic Patch) $L$ $=24.01 \mathrm{~mm}$ to $28.01 \mathrm{~mm}$.

The variation of width length of the patch (Parasitic Patch) $W=31.15 \mathrm{~mm}$ to $35.15 \mathrm{~mm}$.

\section{Discussion of Results}

1) The variation of input impedance with frequency for rectangular dimensions (Patch 1 to Patch 5) is shown in Figures 2(a) to 2(e). It is observed that stacked patch antenna shows dual resonance in which lower resonance frequency increases with increasing patch dimensions frequencies increase with increasing the patch dimensions.

2) The variation of VSWR with frequency for rectangular dimensions (Patch 1 to Patch 5) is shown in Figures 3(a) to 3(e) It is observed that the value of VSWR corresponding to lower resonance frequency is decreases from 1.96 to 1.54 with increasing patch dimensions where as at the upper resonance frequency the value of VSWR is also decrease from 1.66 to 1.47.

3) The variation of resonance frequencies with different dimensions of the antenna (Patch 1 to Patch 5) are shown in the Figure 4, It is observed that both resonance frequencies increase with increasing the patch dimensions.

4) The variation of resonance frequency ratio $f_{2} / f_{1}$ with dimensions of the antenna (Patch 1 to Patch 5) is shown 
in the Figure 5. It is observed that both ratios of resonance frequencies are all most constant with increasing the patch dimensions.

5) The variation of different dimensions of the antenna (Patch 1 to Patch 5) with real part of input impedance is shown in Figure 6. It is observed that the real part of input impedance is decrease from 91.27 to 71.20 at lower resonance frequency and increases as 31.87 to 34.78 with increasing the patch dimensions.

\section{Conclusions}

This paper has investigated the effect of introducing di- mensions variation of parasitic patches on the performance of an electromagnetically coupled stacked rectangular microstrip antenna. The dual-band operation is achieved. It is observed from the experimental result that the resonance frequencies ratio all most constant (2.86 to 2.91 $\mathrm{GHz}$ ) with increasing the patch dimensions. The broad band width is also achieved $45 \%$ of the rectangular microstrip antenna by stacking the patches. Therefore the proposed antenna can be used for mobile communication. where as upper resonance frequency is almost constant with increasing the patch dimensions.

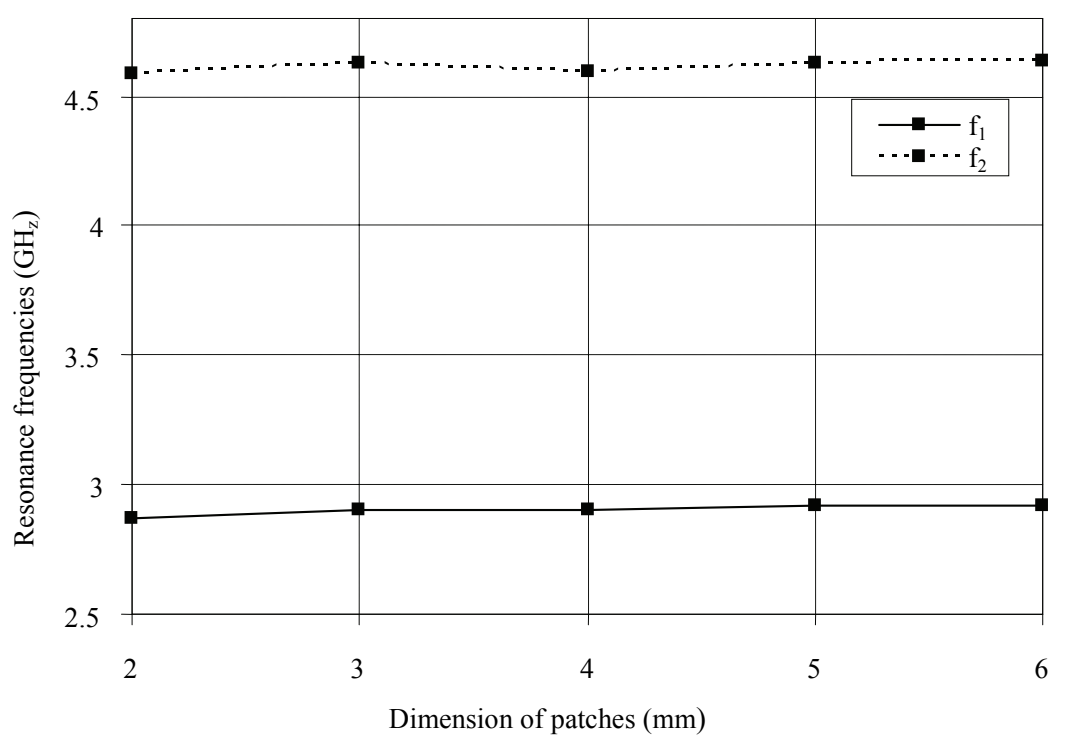

Figure 4. Variation of resonance frequencies with different patch dimensions.

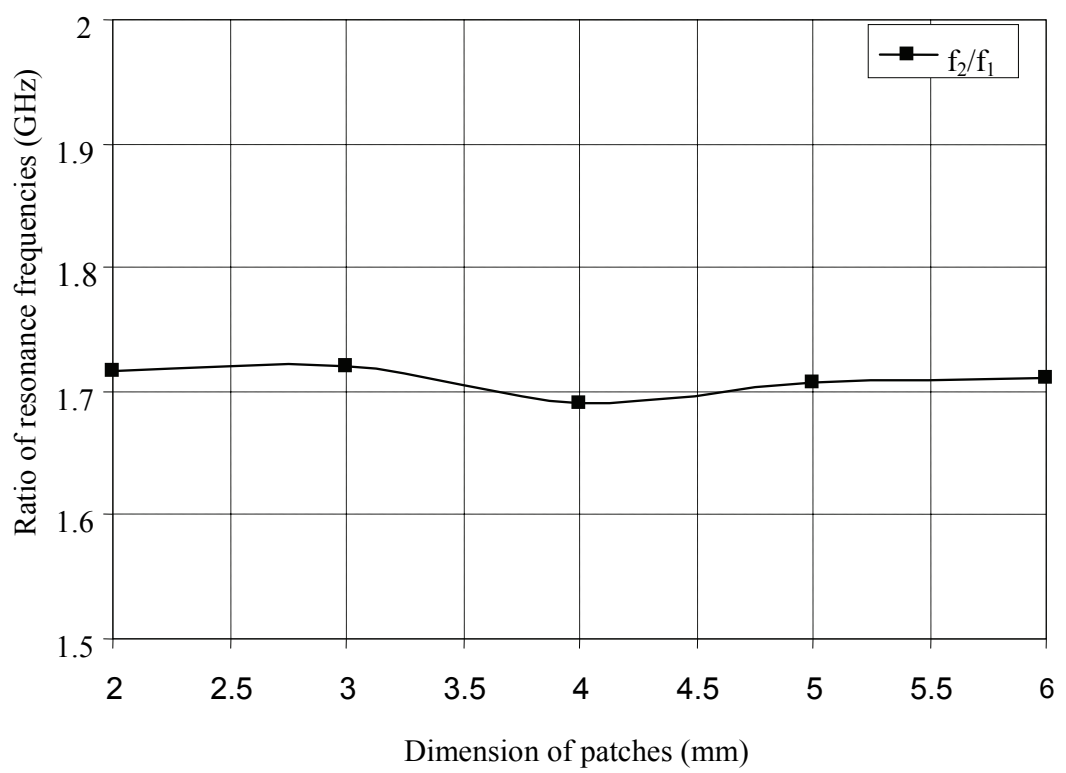

Figure 5. Variation of ratio resonance frequencies with different patch dimensions. 


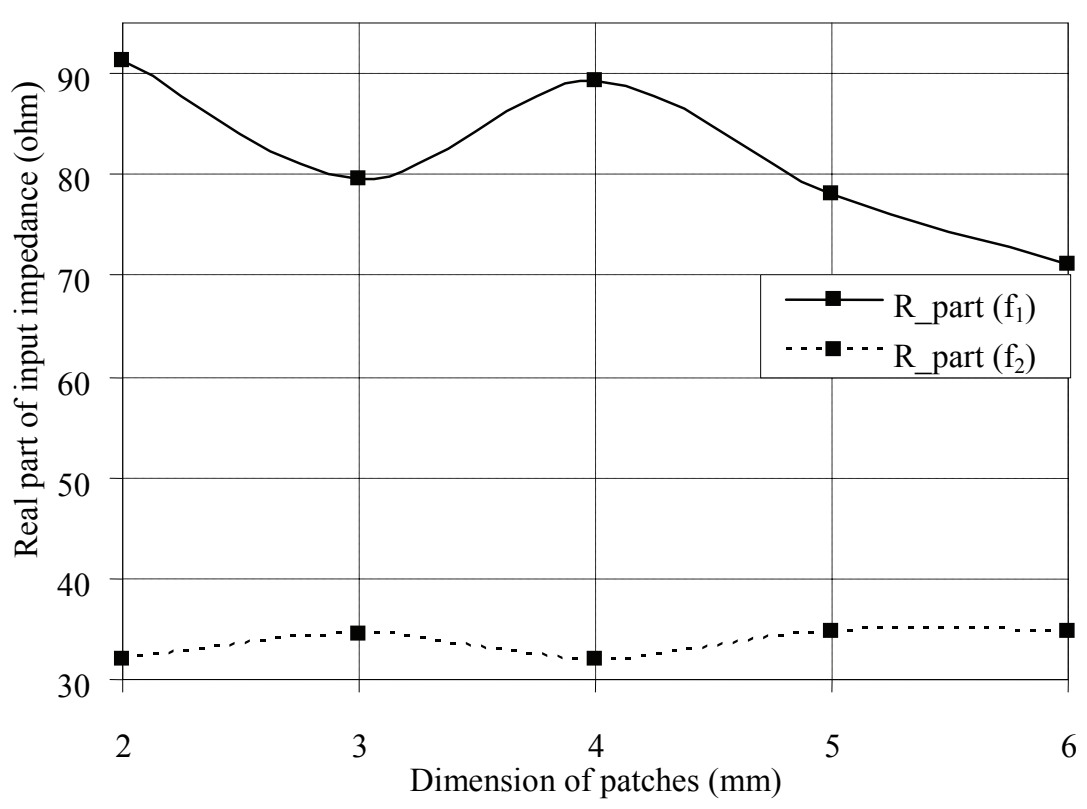

Figure 6. Variations of patch dimensions with real part of input impedance.

\section{Acknowledgments}

The authors would like to thank Professors Arun Kumar and Shri R. K. Malaviya of the Space Application Centre, Indian Space Research Organization Ahmedabad, for providing the measurement facilities.

\section{References}

[1] J. 1. BahI, and P. Bhartia, Microstrip Antennas, Dedham, MA, (USA) Artech House, 1980.

[2] J. R. James, P. S. Hall, and C. Wood, "Microstrip antenna theory and design" IEE Electromagnetic Wave, Series 12 London, U. K. Peter Peregrinus, 1981.

[3] K. C. Gupta, "Recent advance in microstrip antenna," Microwave Journal, No. 27, pp. 50-67, 1984.

[4] S. A. Long and M. D. Walton, "A dual-frequency circular-disc antenna," IEEE Transactions on Antenna and Propagation USA, AP-27, pp. 270-273, 1979.

[5] R. L. Yadava and B. R. Vishvakarma, "Analysis of electromagnetically coupled two-layer elliptical microstrip stacked antennas," International Journal of Electronics, Vol. 87, No. 8, pp. 981-993, 2000.

[6] A. B. Nandgaonkar and S. B. Deosarkar, "Broadband stacked patch antenna for bluetooth applications," Journal of Microwaves, Optoelectronics and Electromagnetic Application, Vol. 8, No. 1, pp. 1-5, 2009.

[7] I. K. Moussa and D. A. E. Mohamed and I. badran, "Analysis of stacked rectangular microstrip antenna," $24^{\text {th }}$ National Radio Science Conference, March 13-15, pp. 1-
10, 2007.

[8] W. Chen, K. F. Lee, and R. O. Lee, "Input impedance of coaxially fed rectangular microstrip antenna on electrically thick substrate," Microwave optical Technology Letters, Vol. 6, No. 6, pp. 387-390, 1993.

[9] F. Pues and R. Van De Capelle, "An impedance matching technique for increasing the bandwidth of microstrip antenna," IEEE Transactions on Antennas Propagation, Vol. 37, pp. 1345-1354, 1989.

[10] J. S. Dahel and K. F. Lee, "A dual-frequency stacked microstrip antenna," IEEE AP-S International Symposium Digest, pp. 308-30-11, 1982.

[11] K. F. Lee, K. Y. Ho, and J. S. Dahele, "Circular-disk microstrip antenna with air gap", IEEE Transactions on Antenna (USA) and Propagation, AP-32, pp. 880-884, 1984.

[12] J. S. Dahele, K. F. Lee, and D. P. Wong, "Dual frequency stacked annular ring microstrip antenna," IEEE Transactions Antennas and Propagation (USA) AP-35, pp. 12811285, 1987.

[13] D. M. Pozar and S. M. Duffy. "A dual-band circular polarized aperture-coupled stacked microstrip antenna for global positioning satellite, "IEEE Transactions on Antennas and Propagation (USA), Vol. 45, No. 11, pp. 1618-1625, 1997.

[14] S. Egashira and E. Nishiyama "Stacked microstrip antenna with wide bandwidth and high gain," IEEE Transactions on Antennas and Propagation (USA) Vol. 44, No. 11, pp. 1533-1534, 1996.

[15] R. E. Harrington, "Time harmonic electromagnetic field," Ms Graw-Hill, New Delhi, 1979. 